\title{
Dynamic channel coding for efficient Motion JPEG2000 video streaming over Mobile Ad-hoc Networks
}

\author{
Max Agueh, J-F Diouris \\ IREENA -Dept Com Num \& RF \\ Rue Christian Pauc-la chantrerie \\ 44306 Nantes cedex 3 - France \\ +33 240-683-237 \\ max.agueh@univ-nantes.fr \\ jean-francois.diouris@univ-nantes.fr
}

\begin{abstract}
In this work, we present a dynamic channel coding scheme for robust transmission of JPEG2000 codestreams over Mobile Adhoc Networks (MANET). The proposed system, based on dynamic Forward Error Correction (FEC) rate allocation allied to layered Unequal Error Protection (UEP), is implemented according to the main recommendations of Wireless JPEG2000 standard final draft. We demonstrate that dynamic FEC rate allocation outperforms a priori FEC rate allocation. Going straightforward we validate the proposed scheme by deriving interesting results from Motion JPEG2000 video streaming over real MANET data traces.
\end{abstract}

This application, developed within the European IST WCAM project, validates wireless JPEG2000 standards recommendations and is a step toward guaranteeing Quality of Service (QoS) in wireless JPEG2000 based data streaming systems.

\section{Keywords}

Forward Error Correction (FEC), layered Unequal Error Protection (UEP) with Reed-Solomon codes, wireless JPEG2000 (JPWL), wireless video streaming, MANET.

\section{INTRODUCTION}

Nowadays, more and more multimedia applications integrate wireless transmission functionalities. Wireless networks are suitable for those types of applications, due to their ease of deployment and because they yield tremendous advantages in terms of mobility of User Equipment (UE). However, wireless networks are subject to a high level of transmission errors because they rely on radio waves whose characteristics are highly dependent of the transmission environment. In wireless video transmission applications like the one considered in this paper and presented in Figure 1, effective data protection is a crucial issue.

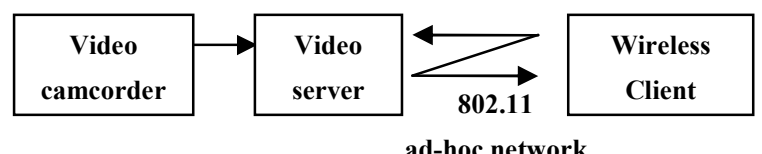

Figure 1: Wireless video streaming system

Permission to make digital or hard copies of all or part of this work for personal or classroom use is granted without fee provided that copies are not made or distributed for profit or commercial advantage and that copies bear this notice and the full citation on the first page. To copy otherwise, to republish, to post on servers or to redistribute to lists, requires prior specific permission and/or a fee. MOBIMEDIA 2007, August 27-29, Nafpaktos, Greece

Copyright (C) 2007 ICST 978-963-06-2670-5

DOI 10.4108/ICST.MOBIMEDIA2007.1852

\author{
Magaye Diop \\ ESP - UCAD \\ BP 5085 \\ Dakar - Senegal \\ $+221825-47-23$ \\ magaye@ucad.sn
}

\author{
F-O Devaux \\ Communications and Remote \\ Sensing Laboratory, FSA/TELE \\ batiment Stevin, place du levant 2 \\ B-1348 Louvain-la-neuve-Belgium \\ +32 10-47-80-72 \\ devaux@tele.ucl.ac.be
}

JPEG2000, the newest image representation standard, addresses this issue firstly by including predefined error resilient tools in his core encoding system (part 1) and going straightforward by defining in its $11^{\text {th }}$ part called wireless JPEG2000 ( JPWL) a set of error resilient techniques to improve the transmission of JPEG2000 codestreams over error-prone wireless channel. In [1], JPWL system description is presented and the performance of its Error Protection Block (EPB) is evaluated. A fully JPEG2000 Part 1 compliant backward compatible error protection scheme was proposed in [2] where a memoryless Binary Symmetric Channel (BSC) is used for simulations. As packets errors arrive in burst in wireless channel the channel model used in [2] is not realistic.

The wireless JPEG2000 system presented in this paper aimed at highlighting the importance of protecting JPEG2000 codestream according to JPWL specifications. Hence, JPWL main ideas such as Forward Error Correction and data interleaving are addressed.

To the best of our knowledge the present work is the first to present results derived from JPEG2000 image/video streaming over realistic wireless channel model (Gilbert-Elliot) and real wireless channel traces.

The paper is arranged as follows. In section 2, an overview of JPEG2000 is presented with a focus on JPWL (JPEG2000 $11^{\text {th }}$ part). Then the proposed JPWL based system is described in section 3 with details on its main modules. One subsection is dedicated to a priori and dynamic protection strategies of JPEG2000 codestreams with Reed-Solomon codes. The problem of FEC rate allocation is discussed in section 4 and in section 5 , experimental results are derived from JPEG2000 frames transmission over real MANET data traces. Finally, some conclusions are provided in section 6 .

\section{JPEG2000 AND WIRELESS JPEG2000 (JPWL) OVERVIEW}

JPEG2000 is the newest image compression standard completing the existing JPEG standard [3]. The interest for JPEG2000 is growing since the Digital Cinema Initiatives (DCI) has selected JPEG2000 for future distribution of motion pictures. Its main characteristics are: lossy or lossless compression modes; resolution, quality and spatial scalability; transmission and progressive image reconstruction; error resilience for low bit rate mobile applications; Region Of Interest (ROI) functionality, etc. 
Part 1 of the standard defines different tools allowing the decoder to detect errors in the transmitted codestream, to select the erroneous part of the code and to synchronise the decoder in order to avoid decoder crash. Even if those tools give a certain level of protection from transmission errors, they become ineffective when the transmission channel experiences high bit error rate. Wireless JPEG2000 (JPEG2000 $11^{\text {th }}$ part) addressed this issue by defining techniques to make JPEG2000 codestream more resilient to transmission errors in wireless systems.

Wireless JPEG (JPWL) which is still in standardization process, specifies error resilience tools such as Forward Error Correction, interleaving, unequal error protection.

In this paper we present a wireless JPEG2000 video streaming system based on the recommendations of JPWL final draft [4]

\section{A WIRELESS JPEG2000 IMAGE/VIDEO STREAMING SYSTEM}

\subsection{System Functionalities}

The functionalities of the proposed JPWL based system are presented in figure 2 . The aim of this system is to efficiently transmit a Motion JPEG2000 (MJ2) video sequence through a wireless channel.

The considered wireless channels are: the simulated Gilbert-Elliot channel model and a channel based on real traces.

\subsubsection{Description}

The input of the JPWL codec is a Motion JPEG2000 (MJ2) file. The JPEG2000 codestreams included in the Motion JPEG2000 file are extracted and indexed.

These indexed codestreams are then transmitted to the JPWL encoder ([4] presents a more accurate description of the JPWL encoder used) which applies FEC at the specified rate and adds the JPWL markers in order to make the codestream compliant to the Wireless JPEG2000 standard. At this stage, the frames are still JPEG2000 part 1 compliant, which means that any JPEG2000 decoder is able to decode them.

To increase the JPWL frames robustness, an interleaving mechanism is processed before each frame transmission through the error-prone channel. This is a recommended mechanism for transmission over wireless channel where errors occur in burst (contiguous long sequence of errors). Thank to the interleaving mechanism the correlation between errors is reduced. The step following the JPWL frames interleaving is the RTP packetisation. In this process, JPEG2000 codestream data or other types of data are incorporated into RTP packets as described in [5]. RTP packets are then transmitted through the wireless channel which is modelled by the Gilbert-Elliot channel model. This channel model will be further presented.

At the decoder side, after the depacketisation process, the JPWL decoder corrects and decodes the received JPWL frames and rebuilds the JPEG2000 frames. At this stage, parameters such as Packet Error Rates ( $P E R$ ) are extracted and give information on the channel state. The decoder sends this information back to the JPWL encoder via the Up link.
The last process of the transmission chain is the evaluation of the Peak Signal to Noise Ratio (PSNR) which measures the distortion between the transmitted and the decoded image/video.

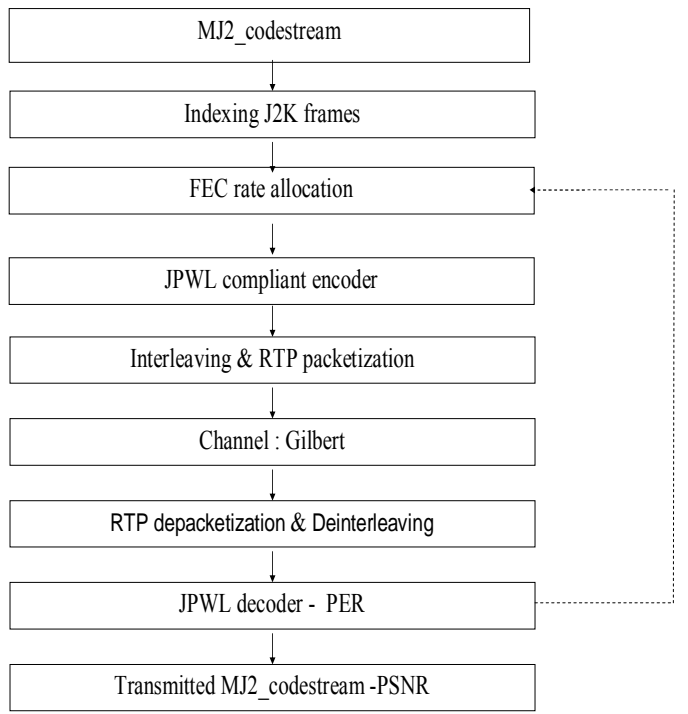

Figure 2: JPWL based system functionalities

\subsubsection{Wireless channel modeling}

When designing the JPWL encoder, a special interest was dedicated to wireless channel modeling. In this paper we do not consider the memoryless Binary Symmetric channel (BSC) because this type of channel model is characterized by uncorrelated error occurrences which are not really representative of real wireless channel. Indeed, wireless channels experience burst errors leading to a correlated repartition of errors. Hence, we demonstrated in [6] that the Gilbert-Elliot model is a good low complex model to emulate burst errors at application level in MANET. This model is based on first order Markov chains and we considered an 8-bit symbol oriented model to emulate the correlated error characteristics of wireless channel.

The Gilbert model was first introduced by Gilbert in [7]. Elliot proposed an extension of the Gilbert model in [8], the last model is commonly known as the Gilbert-Elliot (GE). In the GE model, the wireless channel is modelled to have two states: good and bad. In the good state $(g)$, the channel provides a constant and low symbol error probability $\left(P_{G}\right)$ whereas in the bad state $(b)$, the channel experiences a high symbol error probability $\left(P_{B}\right)$. Hence we have $P_{G}<<P_{B}$ for GE, and $P_{G}=0$ and $P_{B}=1$ for the Gilbert channel, in other word the Gilbert model is a simplified GE model. Therefore, our wireless channel is modelled as a two state Markov process (figure 3).

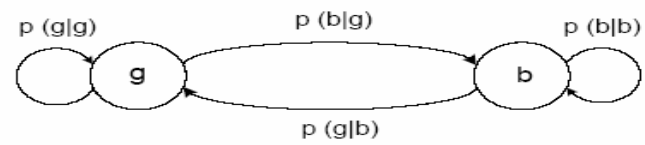

Figure 3: Two-state Markov process scheme 
With this model the channel will produce error bursts. This is because while in bad state the probability of staying in it is greater than the probability of returning to good state. This Markov process is applied for each symbol and based on [9], we derived the Symbol Error Rate ( $S E R$ ) for GE:

$$
\text { SER }=\frac{P_{G} *[1-p(b \mid b)]+P_{B} *[1-p(g \mid g)]}{(1-p(b \mid b)]+1-p(g \mid g)])}
$$

For the Gilbert model, we have $P_{G}=0$ and $P_{B}=1$, so the $S E R$ is given by:

$$
S E R=\frac{[1-p(g \mid g)]}{(1-p(b \mid b)]+1-p(g \mid g)])}
$$

A comprehensive description of the Markov modelling for wireless channel is explained in [10].

\subsection{The protection strategies}

In order to make JPEG2000 codestreams resilient to transmission errors, Unequal Error Protection (UEP) is applied on the codestreams and Forward Error Correction with a priori assignment of Reed-Solomon (RS) codes is used first, this is the a priori strategy and then we consider the proposed dynamic channel coding strategy.

\subsubsection{A priori protection strategy}

In the "a priori protection strategy" we empirically fixed a set of RS codes for each image quality layer. So for an image with L quality layers we chose a set of L Reed-Solomon codes between the RS default codes registered by the JPWL Registration Authority. The most powerful of the L Reed-Solomon codes is assigned to the first layer and the other codes are assigned by decreasing order of power to the other quality layers. By this way all the image content is protected by decreasing order of importance in other words this protection strategy could be seen as a layered unequal error protection with fixed RS codes.

\subsubsection{Dynamic protection strategy}

In the Dynamic strategy the proposed algorithm emulated a the encoder side, uses information like Packet Error Rate ( per ), sent back by the decoder after a previous frame decoding process to evaluate the protection rate for the current frame being processed. By this way, each quality layer protection level is permanently adapted to the transmission condition.

Thank to the dynamic protection strategy and to the proposed algorithm it is now possible to handle the decoding error probability $(P e)$ in the system guaranteeing through a good level of image quality during the transmission. So, even if this protection strategy slightly increases the complexity of the encoder protection module, it brings interesting improvement in terms of image quality and the most important of all it implies the notion of Quality of Service (QoS) in the transmission chain. However, the effectiveness of the 'dynamic protection strategy' strongly relies on the FEC rate allocation scheme.

\section{FEC RATE ALLOCATION PROBLEM}

\subsection{Problem Formalisation and related work}

The problem of FEC allocation can be formulated as follow: given a wireless video streaming system with an available bandwidth of $B_{s}$ (in bit/s) how the JPWL codec could choose the optimal FEC rate to apply to the layers of each frame in order to maximize the overall video quality?

In [4], the JPEG2000 standardisation committee proposed a wireless transmission extension in which Error Sensitivity Descriptors (ESD) give input to the error protection parameters of the transmitted image packets. Up to now, many authors addressed the FEC allocation problem through the angle of a joint sourcechannel coding problem and proposed constrained optimization algorithms to solve the problem. Their algorithms apply Unequal Error Protection (UEP) based on the sensitivity of the JPEG2000 substreams, so in [11][12] as in other similar works, a distortion metric is minimized under a constraint bit rate budget.

In [13], making an analogy between the FEC rate allocation problem and the Multiple-Choice Knapsack Problem (MCKP) leads to the conclusion that both problems are NP-hard. So most of the algorithms proposed in the literature led to exhaustive search among different FEC rate solutions, exponentially increasing their complexity. These algorithms are thus interesting for an offline video streaming but are unpractical for real time applications.

We propose a new, frame based low complex algorithm for FEC rate allocation for efficient real time Motion JPEG2000 video streaming over wireless channel.

\subsection{Proposed algorithm}

Based on Motion JPEG2000 independent constituting frames we designed an algorithm which is build on a new frame oriented approach in the sense that the available bandwidth is considered in terms of symbol per frame instead of in bit/s as it is commonly done in other related work.

The proposed algorithm processed each JPEG2000 frames and derived the FEC rate for each layer deciding at the same time which layer should be transmitted or not in regard to the available bandwidth.

\subsubsection{Basic assumptions and derived analytical metrics}

Let $B_{s}$ be the available bandwidth in bit per second and $B_{a v}$ be the available bandwidth in symbol per frame. As the Forward Error Correction (FEC) is done at the byte level, we have $m=8$ bits per symbol. Fixing the MJPEG2000 sequence rate to $R_{\text {frame }}=24$ frame $/ s$ leads to ${ }_{B_{a v}}=\frac{B_{s}}{m^{*} R_{\text {frame }}}=\frac{B_{s}}{192}$ (in byte / frame $)$

Let $l_{q}$ be the length (in bytes) of the $q$ 'th quality layer data stream. If $R S(n, k)$ is the Reed-Solomon code used for the byte level FEC, a packet is a $n$ bytes stream with $k$ information 
data and $n-k$ redundant data. The code capacity is thus $t=\frac{n-k}{2}$. Though detailing the interleaving process is not in the scope of this paper, it is worth noticing that a good interleaving scheme (which will be further discussed) increases the codestreams robustness to transmission errors.

Let $G_{q}$ be the image $q$ 'th quality layer goodput (useful data after error correction), we have: $G_{q}=\sum_{i=0}^{q} l_{i} * \frac{n_{i}}{k_{i}} *(1-P e)$ $R S\left(n_{i}, k_{i}\right)$ is the RS code used to protect the considered layer.

\subsubsection{Heuristic}

It has been widely observed that the Peak-Signal-to-Noise-Ratio (PSNR) is proportional to the video goodput [14][15][16]. This observation can be extended to JPEG2000 based video streaming system because contrary to temporal schemes such as MPEG-2 and H 264, JPEG2000 encodes a single frame at a time, so errors don't propagate through multiple frames, which increases video goodput. So improving the video quality (when using the PSNR as an objective quality metric) can be achieved by simultaneously maximizing the goodput over all the frames and reducing the decoding error probability.

As the base layer $(q=0)$ is the most important one, it should be protected with the most powerful RS code without exceeding the available bandwidth. Thus, an RS code is used for each quality layer in such a way that for a given bandwidth, the maximum data are correctly transmitted. Let $\mathcal{E}_{0}$ be the maximum decoding error probability that we tolerable in the system, $B_{\text {ness }, i}$ the bandwidth necessary for the transmission of the $i$ 'th layer and $B_{\text {rest }, i}$ the available bandwidth in the system when the $i$ 'th layer is transmitted with the selected FEC rate. We consider $\mathcal{E}_{0}$ as the Quality of Service (QoS) metric so fixing $\mathcal{E}_{0}$ for the base layer and $\varepsilon_{1}=2 * \varepsilon_{0}$ for the enhancement layers is equivalent to fixing a QoS criterion in the system. This criterion is of central importance in our algorithm. As $k_{i}=32$ (cf. JPWL final draft), the FEC rate is obtained by finding the $R S\left(n_{o}, 32\right)$ codes to apply to each layer which maximize the overall goodput and reduce the decoding error probability $(\mathrm{Pe})$. Explicitly, the problem becomes finding the maximal $n_{i}$ such that $P e \leq \varepsilon_{0}$ and the overall goodput $G=G_{0}+\sum_{i=1}^{l} G_{i}$ is maximized.

\subsubsection{Incorrect decoding probability estimation}

In our work we do not consider a cross layer estimation of transmission errors and we fixed the RTP packets payload (1032 byte or symbols for all runs). The RTP Packet Error Rate estimated at the decoder side is used by the FEC allocation algorithm, at the encoder side, to emulate the Gilbert model from which we derived the probability $P e$ that a decoded word is incorrect. In [9], J. R.Yee and E. J.Weldon proposed two methods, combinatorial and recursive, to estimate $P(m, n)$ the probability of $m$ errors in a sequence of $n$ symbols for different error-correcting abilities. We rely on the double recursion method described in this work to estimate $P e$ the decoding error probability: $P_{e}=\sum_{m=t+1}^{n} P(m, n)$

Where $P(m, n)=P_{G}(m, n)+P_{B}(m, n)$

$P_{G}(m, n)$ is the probability of $m$ errors in $n$ transmissions with the channel ending in state $G$ and $P_{B}(m, n)$ is the probability of $m$ errors in $n$ transmissions with the channel ending in state $B$.

\subsubsection{Algorithm}

For frame

$n_{i} \in\{37,38,40, \cdots, 128\}$ (cf JPWL final draft)

Base layer $(q=0)$ :For $n_{i}$

$$
\begin{aligned}
& \text { Evaluate } G_{0}=l_{0} * \frac{n_{i}}{k_{0}} *(1-P e) \\
& B_{\text {ness }, 0}=l_{0} * \frac{n_{i}}{k_{0}} \\
& \text { If }\left(B_{\text {ness }, 0} \leq B_{a v} \& \quad P e \leq \mathcal{E}_{0}\right)
\end{aligned}
$$

then process the following $n_{i}$

$$
\begin{aligned}
& \text { Else } n_{q, o}=n_{i} \\
& B_{r e s t, 0}=B_{a v}-l_{0} * \frac{n_{i}}{k_{0}}
\end{aligned}
$$

End For

Layer $q=l:$ If $B_{\text {rest }, l}=B_{a v}-\left(\sum_{i=1}^{l-1} B_{\text {rest }, i}+B_{\text {rest }, 0}\right) \geq 0$

For $n_{i}$

Evaluate

$$
\begin{aligned}
& G_{l}=G_{l-1}+l_{l} * \frac{n_{i}}{k_{l}} *(1-P e) \\
& B_{n e s s, l}=\sum_{i=1}^{l-1} B_{n e s s, i}+B_{n e s s, 0}
\end{aligned}
$$$$
\text { If }\left(B_{n e s s, l} \leq B_{a v} \& P e \leq \varepsilon_{1}\right)
$$

then process the following $n_{i}$

$$
\text { Else } n_{q, o}=n_{i}
$$

End for

End For 


\subsection{FEC rate estimation}

We used speedway0.j2k ( frame 0 extracted from speedway.mj 2 [17], 288x352, 3 components, 3 layers) to test and validate our algorithm and the available bandwidth is $18 \mathrm{Mbit} / \mathrm{s}$. The use of three layers for FEC estimation is empiric but not restrictive, thus, it could be increased. Figure 4 shows the FEC level $\left(n_{o}\right)$ for the protection of the three layers of the JPEG2000 image for per $=2.4 * 10^{-2}$ and with different Quality of Service level (different $\mathcal{E}_{o}$ ). Each FEC level $\left(n_{o}\right)$ corresponds to a specific $R S\left(n_{o}, 32\right)$ code used for layers protection. As expected, increasing the QoS constraint (decreasing $\mathcal{E}_{o}$ ) leads to high protection level $\left(n_{o}\right)$ for base and enhancement layers. For low $\mathcal{E}_{o}\left(\varepsilon_{o} \leq 10^{-10}\right)$, high QoS constrained system, the protection level is high $\left(n_{o} \geq 80\right)$ for layer 0 et 1 meaning that high error correcting codes are required to achieve the desired Quality of Service (QoS). However high error correcting ability implies high bandwidth consumption leading to non transmission of the last enhancement layer $2\left(n_{o}=0\right)$. Relaxing the constraint on $\mathcal{E}_{o}\left(10^{-5} \leq \varepsilon_{o} \leq 10^{-10}\right)$ leads to reasonable protection level $\left(n_{o}\right)$ selection, yielding simultaneous transmission of all protected layers.

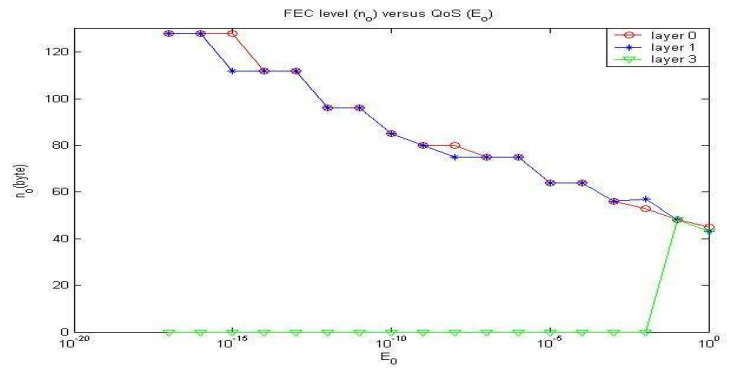

Figure 4: FEC level $\left(n_{o}\right)$ versus $\mathcal{E}_{o}$

It is worth noticing that increasing the number of layers, allows increasing QoS constraint on the most important layers, yielding an improvement of the overall algorithm's performance.

We evaluated in the following section the effectiveness of the dynamic strategy in comparison to the a priori protection.

\section{JPEG2000 VIDEO STREAMING RESULTS}

The interest of this section is to compare the a priori and the dynamic protection strategies and to demonstrate the superiority of the last one. We first show the results achieved when using Gilbert channel models and highlight the practical interest of the dynamic protection strategy by using real wireless channel traces. The video sequence used is the speedway.mj2 [17] containing 200 JPEG2000 frames at an overall quality of $0.2 \mathrm{bpp}$ (bit per pixel) with $0.05 \mathrm{bpp}$ for base layer, $0.1 \mathrm{bpp}$ for the second layer and 0.2 bpp for the third layer.

As error occurrence in the transmission channel is a random process, different runs were made for each simulation and the
Mean Square Error ( $M S E$ ) between the original image ( $\left.I_{o}\right)$ and the decoded image $\left(I_{d}\right)$, is averaged over all the runs in order to have statistically representative metrics. The measured Peak Signal to Noise Ratio (PSNR) is obtained as follows: $\operatorname{MSE}\left(I_{o}, I_{d}\right)=\frac{1}{M \cdot N} \sum_{x=1}^{M} \sum_{y=1}^{N}\left|I_{o}(x, y)-I_{d}(x, y)\right|^{2}$

$$
\overline{M S E}=\frac{M S E}{N_{\text {frames }}} \quad \quad P S N R=10 * \log { }_{10}\left(\frac{255^{2}}{\overline{M S E}}\right)
$$

Where $\overline{M S E}$ is the Mean Square Error over all the $N_{\text {frames }}$ images considered. In the case of Motion JPEG2000 streaming, $N_{\text {frames }}$ represents the 200 JPEG2000 frames constituting the video sequence Each PSNR measure is associated to a decoding rate metric which corresponds to crash estimation on the basis of frames transmission trials.

In a priori protection strategy, the protection rate is $r=5 / 6$ and the selected Reed-Solomon codes used for all image/video streaming tests are: RS $(40,32)$ for base layer, RS $(38,32)$ for layer 2 , RS $(38,32)$ for layer 1

For dynamic protection strategy, we used the algorithm presented in the previous section. Through a wireless client/server application the JPEG2000 frames extracted from the Motion JPEG2000 (MJ2) file are transmitted to the receiver which represents the wireless client, the effectiveness of the JPWL codec is evaluated by computing the PSNR at the output of the system.

The wireless channel is emulated by wireless channel traces [18]. Those traces are a set of loss patterns covering different transmission scenarios (mobile or static). They where generated by connecting two laptops in ad-hoc network using two PCMCIA IEEE $802.11 \mathrm{~b} / \mathrm{g}$ cards (at $2,4 \mathrm{GHz}$ ).

The video sequence is streamed over the generated loss patterns. Each pattern corresponds to a specific Carrier to Noise ratio $\frac{C}{N}$ $\frac{C}{N}$ is the ratio between the desired signal and the total received noise power). The considered loss patterns had a $\frac{C}{N}$ varying between $11 \mathrm{~dB}$ and $20 \mathrm{~dB}$ corresponding to Bit Error Rate between 0.0001 and 0.025 . The used mode at the physical layer of the wireless link is the mode 4 where the modulation is QPSK, the coding rate is $3 / 4$ and the Nominal Data Rate $R_{\text {Nominal }}$ is $18 \mathrm{Mbit} / \mathrm{s}$.

Table 1 shows that PSNR is almost the same $(42 \mathrm{~dB})$ for a priori and dynamic protection. Moreover the worst case PSNR is the same in both strategies leading to the interesting conclusion that in our scenario all well decoded frames have a good and almost fixed quality ( $P S N R \approx 42 d B$ ). When considering the successful decoding rate in figure 5 , we noticed that for channels experiencing $P E R \leq 8^{*} 10^{-1}$ both strategies, a priori and dynamic perform the same way in terms of decoding rate. 
Table 1: Well decoded JPEG2000 frames mean PSNR

\begin{tabular}{|l|c|c|c|}
\hline \multicolumn{1}{|l|}{ PER } & $5.1 * 10^{-3}$ & $6.13 * 10^{-2}$ & $2.662 * 10^{-1}$ \\
\hline A priori FEC & 42.10 & 42.07 & 42.07 \\
\hline PSNR(dB) & 41,39 & 41,39 & 41,39 \\
\hline Worst case PSNR & 42.07 & 42.07 & 42.07 \\
\hline Dynamic FEC & 41,39 & 41,39 & 41,39 \\
\hline PSNR(dB)
\end{tabular}

This is due to the fact that the wireless channel considered experiments few transmission errors so channel coding do not significantly impacts JPEG2000 decoder performance. For $8 * 10^{-1} \leq P E R \leq 10^{-1}$ the dynamic protection strategy increases the successful decoding rate $5 \%$ more than a priori protection.

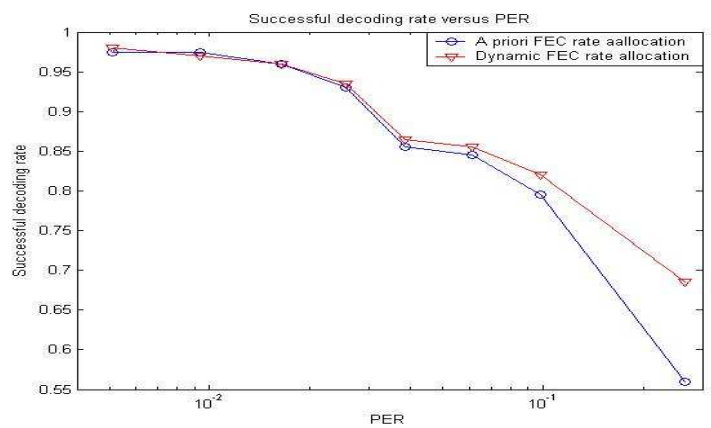

Figure 5: Successful decoding rate versus PER

For $P E R \geq 10^{-1}$ dynamic FEC rate allocation outperforms a priori FEC strategy by a $10 \%$ supplementary increase of the successful decoding rate.

\section{CONCLUSION}

In this paper, a JPWL compliant system based on FEC rate allocation scheme for robust transmission of JPEG2000 images/video over MANET is presented.

We presented two protection strategies, a priori and dynamic FEC rate allocation based on layered Unequal Error Protection (UEP). We discussed the problem of FEC rate allocation and proposed a low complex algorithm for dynamic FEC rate allocation. Through an application of Motion JPEG2000 video streaming over real MANET data traces we demonstrated that dynamic FEC rate allocation outperforms a priori protection strategy and thanks to the proposed algorithm we handled the decoding error probability in the system under the bandwidth constraint. Summarizing we can say that the proposed JPWL compliant system based on dynamic FEC rate allocation with layered Unequal Error Protection, is a valid foundation to accomplish efficient and reliable JPEG2000 based wireless multimedia transmissions.

\section{REFERENCES}

[1] Frederic Dufaux and Didier Nicholson." JPWL: JPEG2000 for Wireless Applications "Proceeding of SPIE - Vol 5558 Applications of Digital Image Processing XXVII, Andrew G. Tescher, Editor, , pp. 309-318, November 2004

[2] D. Nicholson, C. Lamy-Bergot, X. Naturel and C. Poulliat, "JPEG2000 backward compatible error protection with Reed-Solomon codes," IEEE Transactions on Consumer Electronics, vol. 49, n. 4, pp.855-860, Nov. 2003.

[3] D.S. Taubman et M.W. Marcellin. "JPEG2000 Image Compression Fundamentals, Standards and Practice" Kluwer Academic Publishers, The Netherlands 2001

[4] JPEG2000 part 11 Final Draft International Standard, ISO/IEC JTC 1/SC 29/WG 1 N3797

[5] H. Schulzrinne, S. Casner, R. Frederick, V. Jacobson. "RTP: A Transport Protocol for Real-Time Applications". draft-ietfavt-rtp-new-12.txt, March 2003.

[6] M. Agueh, J-F Diouris “ Application level channel modelling in wireless network: exemple of JPEG2000 images/video streaming over wireless channel" - IRAMUS Workshop Val Thorens, France, 25-26 Jan 2007

[7] E. N. Gilbert, "Capacity of a burst noise channel," Bell Syst. Tech. J., vol. 398, pp. 1253-1266, Sept 1960

[8] O.Elliot, "Estimates of error rates for codes on burst-noise channel," Bell Syst.Tech. J., vol. 42, pp. 1977-1997, Sept 1963

[9] J. R.Yee, E. J.Weldon, "Evaluation of the performance of errorcorrecting codes on a Gilbert channel", in IEEE trans. commun. vol. $43, \mathrm{n}^{\circ} 8$, pp. 2316-2323, 1995

[10] Julio Arauz, prashant Krisnamurthy. "Markov Modeling of 802.11 channels" in Vehicular Technology Conférence, 2003.VTC 2003-Fall. 2003 IEEE 58 ${ }^{\text {th }}$. Vol.2, pp 771- 775, 6-9 Oct. 2003

[11] C. Poulliat, I. Fijalkow, and D. Declercq. "Scalable image transmission using UEP optimized LDPC codes". In ISIVC'04, Brest, France, Jul 04

[12] Nikolaos Thomos, Nikolaos V. Boulgouris, and Michael G. Strintzis." Wireless transmission of images using JPEG2000". In ICIP'04, Singapore, October 2004.

[13] A. E. Mohr. Bit Allocation in Sub-linear Time and the MultipleChoice Knapsack Problem" In Data Comp. Conf., Snao Bird, Utah, USA, March 2002. IEEE Computer Society Press

[14] S. Gringeri, S. Iren, and R. Egorov, "Transmission of MPEG-4 video over the Internet" Proc. IEEE Int. Conf. Multimedia and Expo, vol. 3,pp. 1767-1770, July/Aug. 2000.

[15] O. Verscheure, P. Frossard, and M. Hamdi, "Joint impact of MPEG2 encoding rate and Mcell losses on video quality," Proc. IEEE Global Telecom. Conf., vol. 1, pp. 71-76, Nov. 1998.

[16] Y. Wang and Q.-F. Zhu, "Error control and concealment for video communication: A review" Proc. IEEE, v. 86, p. 974-997, May 98.

[17] Speedway video sequences have been generated by UCL, in the context of the project MODEST. Available: www.tele.ucl.ac.be/PROJECTS/MODEST/sequences.htm

[18] Loss patterns acquired during the WCAM Annecy 2004 measurement campaigns IST-2003-507204 WCAM "Wireless Cameras and Audio-Visual Seamless Networking”. Available: http://www.ist-wcam.org 